\title{
Partonic Equation of State in Relativistic Heavy Ion Collisions
}

\author{
N. $\mathrm{Xu}$ \\ Nuclear Science Division, Lawrence Berkeley National Laboratory \\ 1 Cyclotron Road, Berkeley, CA 94720, USA
}

Received on 20 October, 2006

\begin{abstract}
Recent results on chemical freeze-out, elliptic flow $v_{2}$ and multi-strange hadron transverse momentum distributions are discussed and compared with model predictions. The idea of heavy flavor collectivity and light flavor thermalization is presented in the light of recent heavy flavor measurement at RHIC.
\end{abstract}

Keywords: Flow; EOS

\section{INTRODUCTION}

The goal of the RHIC and the future LHC programs is to identify and study the matter with partonic degrees of freedom and determine the phase structure of the QCD phase diagram $[1,2]$. In high-energy nuclear collisions, due to large initial density and frequent re-scatterings, collective motion develop [3]. Depends on both the duration and the strength of the frequent scatterings, local thermalization might be reached in central collisions. Whence the equilibrium limit is reached, the collective motion approaches its maximum. Therefore, the information on the equation of state can be extract in analyzing the flow distributions. Since the strength of flow depends on the nature of the interactions, different hadrons that freezeout at different stages of the collision will carry different flow. For example, heavy flavor and multi-strange hadrons freezeout early [4] so they are powerful tools for studying evolution at the early partonic stage in high-energy nuclear collisions [5].

In this paper, we will focus on bulk production. Those who are interested in the physics issues of initial conditions and high $p_{T}$ phenomena are referred to Refs. [6-8] and references therein. We will first discuss the recent results on partonic collectivity observed at RHIC. Chemical freeze-out and transverse momentum distributions will be compared with model predictions. Later, we discuss the heavy flavor results. The connection between heavy flavor collectivity and light flavor thermalization will be address. A brief summary will be presented at the end.

\section{THE QCD PHASE DIAGRAM}

A sketch of a QCD phase diagram is shown in Fig. 1: temperature versus baryon chemical potentials. The center of mass energies and the corresponding accelerators are also indicated on the plot. In this paper, we will focus on the region relevant to the high-energy nuclear collisions. For those interested in the properties of very high baryon density, we refer the to Ref. [9]. As can be seen, a smaller net-baryon density region is explored in collisions at higher beam energies. In that region, the temperature is almost constant at $T=165 \mathrm{MeV}$. All data points, extracted at the moment of chemical freeze-out, are from Refs. $[10,11]$. The excellent fit obtained to the measured ratios in the figure, including sta-

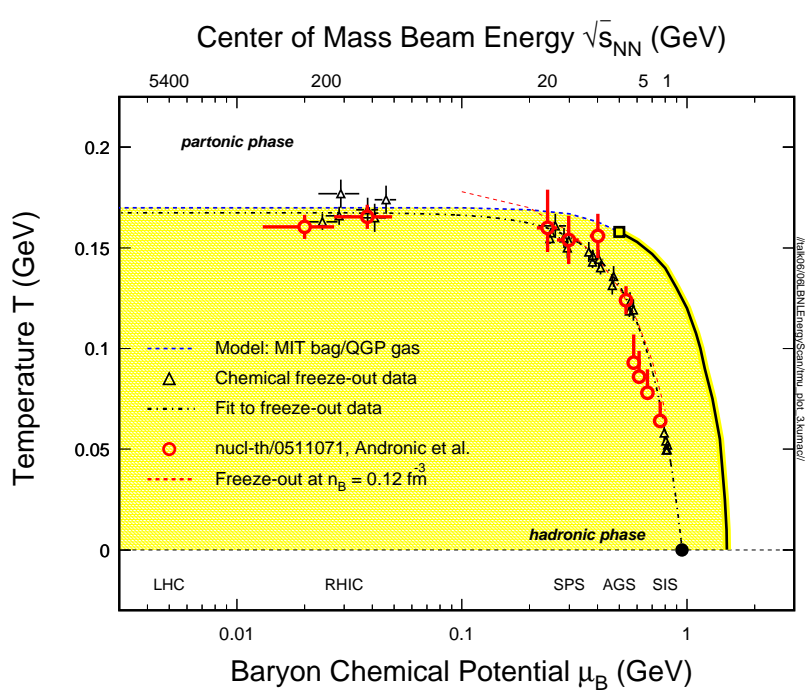

FIG. 1: The QCD phase diagram: Temperature versus baryon chemical potential. The corresponding center of mass energy for collisions is shown at the top of the plot. Circles and triangles are thermal model fit results $[10,11]$. Dot-dashed line represents the parametrization of the data [11]. The first-order phase boundary is indicated by the solid-line and the open square indicates the possible tri-critical point [9]. Dashed-line indicates a possible cross-over.

ble and long-lived hadrons through multi-strange baryons, is consistent with the light flavors, $u, d$, and $s$, having reached chemical equilibrium for central collisions at $T_{c h}=160 \pm 10$ $\mathrm{MeV}[10,12]$. The deviations of the short-lived resonance yields are the results of hadronic rescatterings after the chemical freeze-out.

The temperature deduced from the fits is essentially equal to the critical value of critical temperature from a Lattice QCD calculation. Therefore the deduced value of $T_{c h}$ could be considered as both the hadronization and a lower limit of the partonic thermalization temperature. Now the questions are: How is the energy partitioned between collective motion and random thermal motion in the final state hadrons? What is the partonic EOS? Where is the phase boundary in the QCD phase diagram?

\section{ELLIPTIC FLOW AND COALESCENCE}

In non-central collisions the configuration space anisotropy is converted into a momentum space anisotropy [3]. The col- 
lision dynamics will determine the degree of the transformation. For a symmetric system, like $A u+A u$, a good parametrization of the anisotropy is the second Fourier coefficient $v_{2}$ [13]. At RHIC strong collective flow has been observed for all measured hadrons indicating strongly interacting system created in the collisions.

$v_{2}$ distributions for identified hadrons, like $\pi, K, K_{S}^{0} p, \Lambda$, $\Xi, \Omega$ are shown in Fig. 2. Hydrodynamic model calculations are shown as dashed lines and the fit results for quark number scaling are shown by the dot-dashed lines. At the lower $p_{T}$ region, $p_{T} \leq 2 \mathrm{GeV} / \mathrm{c}$, the value of $v_{2}$ is inversely proportional to the mass of the hadron - characteristic of hydrodynamic collective motion at work, see Fig. 2 (a) and (b). At the intermediate $p_{T}$ region, the dependence is different. Instead of the mass dependence, there seems to be a hadron type dependence. This feature can be seen clearly in Fig. 2 (b).

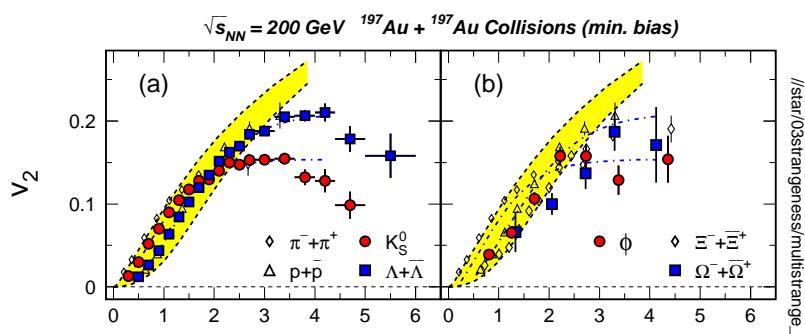

Transverse momentum $\mathrm{p}_{\mathrm{T}}(\mathrm{GeV} / \mathrm{c})$

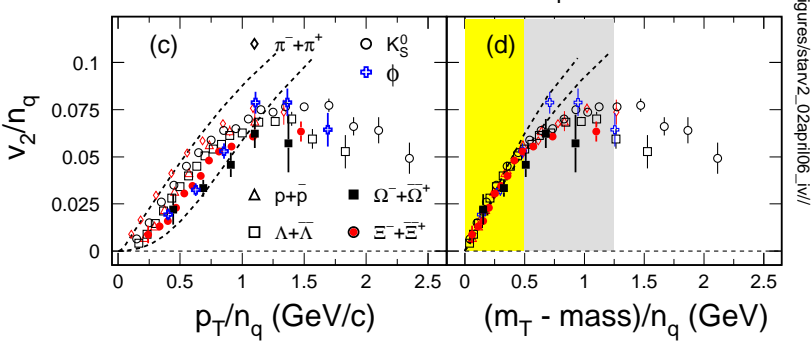

FIG. 2: Identified hadron anisotropy parameters as a function of transverse momentum $p_{T}$ (a) and (b); scaled $p_{T} / n_{q}$ and scaled transverse kinetic energy $\left(m_{T}-\right.$ mass $) / n_{q}(\mathrm{~d}) . n_{q}$ is the number of quark in a given hadron. All data are from minimum biased $A u+A u$ collisions at $\sqrt{s_{\mathrm{NN}}}=200 \mathrm{GeV}$. Dashed-lines are from hydrodynamic calculations [14] and dot-dashed lines are from a number of quark scaling fit [15].

The most fascinating candidate is the $\phi$ meson, whose mass is close to that of $p$ and $\Lambda$. The $v_{2}$ distribution, circles in Fig. 2(b), is the same as the $v_{2}$ for $\pi$ and $K$ ! It is known that the $\phi$ meson does not participate as strongly as others in hadronic interactions. $\phi$ mesons also cannot be formed via the coalescence-like $K^{+}+K^{-}$process [16] in high energy collisions. Thus the observed strong $v_{2}$ must have been developed before hadronization. This observation together with the $\Omega v_{2}$ results provide clear evidence for partonic collectivity [12].

In order to demonstrate the scaling properties of $v_{2}$, we have performed the following transformations: (i) scale the measured $v_{2}$ by the number of valence quarks in a given hadron. For mesons and baryons they are $n_{q}=2$ and 3 , respectively. The $p_{T}$ is also scaled with the same $n_{q}$. The results are shown in Fig. 2 (c). To include the effect of the collective motion, we further transfer the $p_{T}$ to the transverse kinetic energy $m_{T}-$ mass scaled by $n_{q}$. The results are shown in Fig. 2 (d). All of the hadron $v_{2}$ are scaled nicely up to $\left(m_{T}-\right.$ mass $) / \mathrm{n}_{\mathrm{q}} \sim 1.2$. Hydrodynamic calculations [14], shown as dashed-line in the plot, are also scaled in the low $p_{T}$ region. At high $p_{T}$, the values of $v_{2}$ seem to fall.

One might ask why do we care about the scaling properties? In this case, the scaling tells us that prior to hadronization, quarks already acquired the collective motion $v_{2}$ and that when they coalesced, the $v_{2}$ was passed to the newly formed hadrons. Again this observation indicates partonic collectivity. Furthermore, since collectivity requires a correlation at a scale much larger than the size of a given nucleon, the observed partonic collectivity implies deconfinement in such high-energy nuclear collisions.

The coalescence or recombination mechanism for hadronization could be a common process for hadronization even for elementary collisions. For example, in $e+e^{+}$collisions, in string picture, hadrons are formed via 'coalescence' of quarks from the same string. In high-energy nuclear collisions the parton density is much higher than that in elementary collisions, hadrons can be formed, in principle, via 'coalescence' of quarks from difference strings. The patonic collectivity, therefore, will be manifested in the observed hadron momentum distributions.

However, a word of caution is called for because, a hadronic transport model calculations [17] also indicate a particle type dependence of $v_{2}$. While the experimental observation is unique, the explanation might not be. In transport model calculations the overall strength of $v_{2}$ is much less than in the data. This is another hint for partonic interactions at the early stage of the collisions at RHIC. Further tests of the deconfinement plus recombination hypothesis are necessary with high precision $v_{2}$ measurements of resonances like $K^{*}, \rho, \Lambda^{*}$, and $\Xi^{*}[17]$.

\section{HADRON TRANSVERSE MOMENTUM DISTRIBUTIONS}

At RHIC, many hadron transverse momentum distributions, from $\pi$ (contains only $u, d$ quarks) to $\Omega$ (has only $s$ quarks), have been measured with high precision [12]. Recent results on multi-strange hadron $(\phi, \Xi, \Omega)[16,18]$ and heavy flavor $(J / \psi)$ [19] transverse momentum distributions $\left(m_{T}=\sqrt{p_{T}^{2}-\mathrm{mass}^{2}}\right)$ are shown in Fig. 3 left-plot . The exponential fit $\left(f \sim \exp \left(-m_{T} / T\right)\right)$ results are also shown as lines. These data are all from $\sqrt{s_{\mathrm{NN}}}=200 \mathrm{GeV}$ central $A u+A u$ collisions at RHIC. For comparison, the exponential slope parameters of all measured hadrons are summarize in the right-plot of the figure. Results from collisions at the RHIC are shown as dots while the results from the SPS [4, 22] are shown as triangles.

At both collision energies, for those copiously produced hadrons $\pi, K$, and $p$, their mass dependence is much stronger than multi-strange and heavy flavor hadrons indicating a stronger flow for these hadrons. A systematic analysis show the collective velocity of $0.4 c$ and $0.6 c$ are researched for central collisions at the SPS and RHIC $[12,22]$, respectively. For 
multi-strange and heavy flavor hadrons, on the other hand, they show different trends at different collision energy. At the SPS, the values of the slope parameter is about $250 \mathrm{MeV}$ and there is no visible mass dependent.

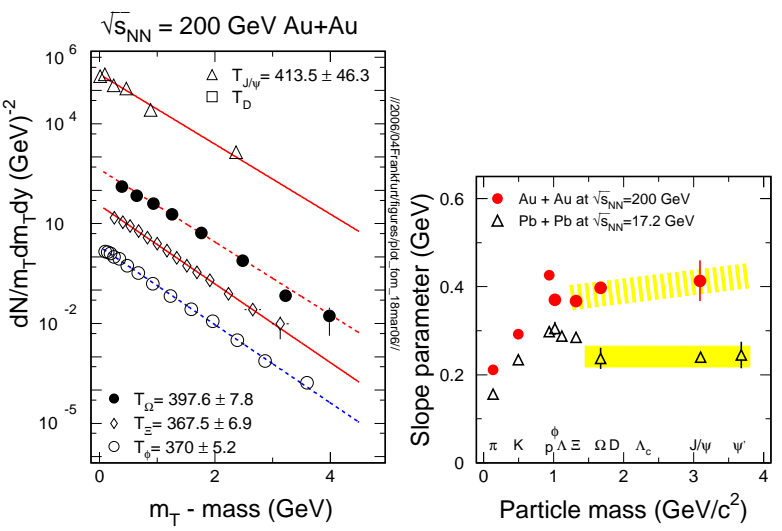

FIG. 3: Left: mid-rapidity transverse momentum distributions for $\phi$ (open circles), $\Xi$ (open diamonds), $\Omega$ (dots) $[16,18]$ and $J / \psi$ (triangles) [19]. Exponential fit results are shown as lines. Right: Inverse slope parameters versus hadron mass from central collisions at the SPS (open triangles) and RHIC (dots).

The lack of mass dependent was explained by the null partonic collectivity in collisions at the SPS. On the other hand, a clear mass dependent in the slope parameter, extracted from collisions at RHIC, is shown in the right plot in Fig. 3. The value of the slope parameter increases from $\phi, \Xi$ to $\Omega$ and to $J / \psi$, demonstrating the development of the partonic collectivity in collisions at RHIC. This observation is perfectly compatible with the strong elliptical flow $v_{2}$ and the numberof-quark-scaling [12] discussed in the earlier section.

We fitted the the mass dependence to the equation: $T=$ $E_{\text {thermal }}+C \cdot \operatorname{mass} \cdot\left\langle\beta_{\mathrm{T}}^{\mathrm{p}}\right\rangle^{2}$ and the resulting partonic collective velocity, for central $A u+A u$ collisions at RHIC, is $\left\langle\beta_{T}^{p}\right\rangle \geq$ $0.2 c$. The uncertainty is in the order of $25 \%$ and more precise determination of the velocity can be obtained with the understanding of the cause of the pre-equilibrium velocity field [20]. As mentioned earlier, the chemical freeze-out temperature seems coincide with the LGT predicted critical temperature [1] meaning: $T_{C} \sim T_{h} \sim T_{\text {chem }}$. Then the hadronization parameters at RHIC are: $T=160 \pm 10 \mathrm{MeV}$ and $\left\langle\beta_{T}^{p}\right\rangle \geq$ $0.2 c$. Note that the temperature parameter is considerably higher than that at the kinetic freeze-out while the velocity parameter is much lower.

\section{SUMMARY}

Integrated hadrons yield ratios are consistent with the chemical equilibration of light flavors including $u, d$, and $s$ quarks at a temperature of $T_{c h}=160 \mathrm{MeV}$ at RHIC. This value is coincide with the critical temperature for the phase transition from hadronic matter to partonic matter. Multi-strange hadron transverse spectra allow us to deduce the partonic collective velocity as $\left\langle\beta_{T}^{p}\right\rangle \geq 0.2 c$. In order to determine the nature of equilibrium at the early stage of the high-energy nuclear collisions, one must measure the collectivity of heavy flavor hadrons.

\section{Acknowledgments}

We are grateful for many enlightening discussions with Drs. S. Esumi, H.G. Ritter, and K. Schweda. This work has been supported by the U.S. Department of Energy under Contract No. DE-AC03-76SF00098.
[1] F. Karsch, Nucl. Phys. A 698, 199c (2002)

[2] Science, 312, 14 April, 2006; http://www.sciencemag.org.

[3] W. Reisdorf and H.G. Ritter, Ann. Rev. Nucl. Part. Sci. 47, 663 (1997) and references therein.

[4] H. van Hecke, H. Sorge, and N. Xu, Phys. Rev. Lett., 81, 5764 (1998).

[5] N. Xu and M. Kaneta, Nucl. Phys. A 698, 306c (2001).

[6] L. McLerran, these proceedings.

[7] A. Suaide, these proceedings.

[8] M. Gyulassy, I. Vitev, X.N. Wang, and B. Zhang, in Quark Gluon Plasma 3, eds. R.C. Hwa and X.N. Wang, World Scientific Publishing and references therein; nucl-th/0302077.

[9] M.A. Stephanov, Prog. Theor. Phys. Suppl. 153, 139 (2004); hep-ph/0402115.

[10] P. Braun-Munzinger, K. Redlich, and J. Stachel, in Quark Gluon Plasma 3, eds. R.C. Hwa and X.N. Wang, World Scientific Publishing and references therein; nucl-th/0304013 and A. Andronic, P. Braun-Munzinger, and J. Stachel, Nucl. Phys. A 772, 167 (2006); nucl-th/0511071.

[11] J. Cleymans, H. Oeschler, K. Redlich, and S. Wheaton, Phys. Rev. C 73, 034905 (2006); hep-ph/0511094.

[12] J. Adams et al., (STAR Collaboration), Nucl. Phys. A 757, 102
(2005).

[13] S. Voloshin and Y. Zhang, Z. Phys. C 71, 665 (1996); A.M. Poskanzer and S. Voloshin, Phys. Rev. C 58, 1671 (1998).

[14] P. Huovinen, P. Kolb, U. Heinz, P.V. Ruuskanen, and S. Voloshin, Phys. Lett. B 503, 58 (2001).

[15] X. Dong, S. Esumi, P. Sorensen, N. Xu, and Z. Xu, Phys. Lett. B 597, 328 (2004).

[16] J. Adams et al., (STAR Collaboration), Phys. Lett. B 612, 181 (2005) and S. Blyth et al., (STAR Collaboration), proceedings of International Conference on Strangeness in Quark Matter, Los Angeles, California, 26 - 31 March 2006; nucl-ex/0608019.

[17] Y. Lu, et al., J. of Phys. G: Nucl. Part. Phys. 32, 1121 (2006); nucl-th/0602009.

[18] J. Adams et al., (STAR Collaboration), nucl-ex/0606014.

[19] H. Buesching and T. Gunji, QM05 conference, August, 2005.

[20] P. Kolb and R. Rapp, Phys. Rev. C 67, 044903 (2003).

[21] J. Adams et al., (STAR Collaboration), Phys. Rev. Lett. 95, 122301 (2005); nucl-exp/0504022.

[22] I.G. Bearden et al., (NA44 Collaboration), Phys. Rev. Lett. 78 2080 (1997). 